\title{
UniDoodle: A Multi-Platform Smart Device Student Response System - Evaluated in an Engineering Mathematics Classroom
}

\author{
Séamus McLoone, Dept. of Electronic Engineering, Maynooth University, Maynooth, Ireland. \\ Email: seamus.mcloone@nuim.ie \\ Christine Kelly, Dept. of Electronic Engineering, Maynooth University, Maynooth, Ireland. \\ Conor Brennan, School of Electronic Engineering, Dublin City University, Dublin, Ireland. \\ Catriona NiShe, School of Mathematical Sciences, Dublin City University, Dublin, Ireland.
}

\begin{abstract}
Most of the existing student response systems, such as clickers, have limited input capabilities, typically only offering students a multiple-choice selection. In some instances, students can input a numerical or textual response. However, mathematical equations, diagrams, etc. are all beyond the capabilities of such systems. This paper proposes and presents a novel multi-platform smart device-based student response system, called UniDoodle, that allows for a more generic and flexible input. This system consists of a student application that allows for freeform input through sketching capabilities, a lecturer application that allows easy viewing of multiple sketch-based responses and a cloud-based service for co-ordinating between these two applications. In essence, students can now respond to a question posed by the lecturer using sketches and, hence, mathematical equations, circuit diagrams, graphs, etc. are all possible on the UniDoodle system. In addition, the lecturer can now gain a richer and more useful insight to the students' understanding of the relevant material. This paper also evaluates the UniDoodle system in a large class of first year Engineering Mathematics students. Details of the UniDoodle system, the evaluation process and the feedback obtained are all presented within.
\end{abstract}

Keywords: student response systems, classroom response systems, formative assessment, technology in the classroom.

\section{Introduction}

Student response systems (SRSs) are slowly becoming more commonplace in the classroom today. Such systems allow students to respond to in-class questions in real-time, in a fast and efficient manner (Fies and Marshall, 2006). These systems exist under many different guises in the research literature, including clickers (Barber and Njus, 2007), classroom response systems (Roschelle et al., 2004), audience responses systems (Ramesh, 2011, Miller et al., 2003), clicker assessment and feedback technology (Han and Finkelstein, 2013), electronic voting systems (Retkute, 2009) and voting machines (Reay et al., 2005). The literature also clearly outlines the many numerous pedagogical benefits that the use of SRSs entails. These include improved classroom interaction, improved student learning, improved motivation, better retention of material and better attendance (Blasco-Arcas, et al., 2013, Bruff, 2009, Caldwell, 2007, Moredich and Moore, 2007, Rowlett, 2010). In addition, action learning activities and classroom assessment techniques (Angelo and Cross, 1993) are easier implemented through the use of SRSs (Sarason and Banbury, 2004, McLoone et al., 2015).

However, to date, these systems only allow for limited input capabilities, whereby students are required to select an answer from a given set of possible answers, i.e. a multiple-choice selection. In some cases, students can input a numerical or a textual response. Either way, mathematical 
equations, circuit diagrams, sinusoidal responses, annotations of diagrams, etc. are all beyond the capabilities of existing SRSs.

This lack of freeform input is a significant drawback for the Science, Technology, Engineering and Mathematics (STEM) disciplines where writing equations, drawing circuits and sketching diagrams are important aspects of the student learning experience. For example, consider the solving of an algebraic equation, the designing of a circuit, the sketching of a mathematical function, etc. The list of such examples is endless and it is very important that students of STEM disciplines, in particular, can carry out such fundamental processes and methodology. In order to capture the real-time feedback of the students' grasp of this information it is necessary for a SRS to facilitate freeform input.

Here, we propose the use of a novel multi-platform smart device-based student response system, called UniDoodle, that allows for a more generic and flexible input. Students can now respond to a question posed by the lecturer using sketches and, hence, mathematical equations, circuit diagrams, graphs, etc. are all possible with this system. In addition, the lecturer can access a richer and more useful insight to the students' understanding of the relevant material. This new system operates on any device that supports either the iOS (iPads, iPhones) or Android (smart phones and tablets) operating system.

The UniDoodle system was evaluated by a large class of first year Engineering Mathematics students in the School of Electronic Engineering in Dublin City University (DCU). Both student and lecturer feedback was obtained at the end of the evaluation period. The evaluation process and a summary of the feedback obtained will be outlined in later sections.

\section{UniDoodle - a brief overview}

UniDoodle is a recently developed, multi-platform, smart device based student response system that provides a freeform-style input using sketch capabilities. It builds on previous work in the area by the lead author (McLoone et al., 2015), and currently operates on all devices running either iOS or Android. This system consists of a student application that allows for freeform input, through sketching capabilities, a lecturer application that allows easy viewing and editing of multiple sketchbased responses (see Figure 1) and a Google App Engine cloud-based service for co-ordinating between these two applications.

Overall, the system works as follows - firstly, the teacher poses a question in class (this can be a pre-prepared question in the form of a template or a new on-the-spot question); secondly the students receive this question (in the case of a template question) on their device and can now respond appropriately using the in-built sketch capabilities (equations, diagrams, graphs, and annotations are all possible); thirdly, the teacher receives the student responses in a neat and concise format on their own device (typically a tablet); and lastly the teacher can point out and respond to any obvious errors that students may have made. In addition, the student responses can be viewed via the overhead projector allowing all students to see all the submitted responses. This offers students a level of peer learning as they can now see where other students are making mistakes, if any, and what those mistakes are.

Several new and important features exist in UniDoodle in comparison with the work carried out by McLoone et al. (2015). Firstly, it supports the preparation of questions in advance of class through the use of templates. Teachers can now pre-prepare questions and load them on the cloud-based database. Furthermore, templates can be arranged into various folders (see Figure 2). For 
example, a teacher could have a different folder of questions for each subject or class they teach. Secondly, diagrams can be created on a PC using any suitable drawing package, saved in any standard image format, and uploaded to the database. This allows for more detailed and precise diagrams to be used as the basis of questions. Finally, UniDoodle includes a filter feature that allows the teacher to remove any undesirable student responses. This quick and easy to use feature means that unwanted images can be deleted before the responses are shown live to the whole class.
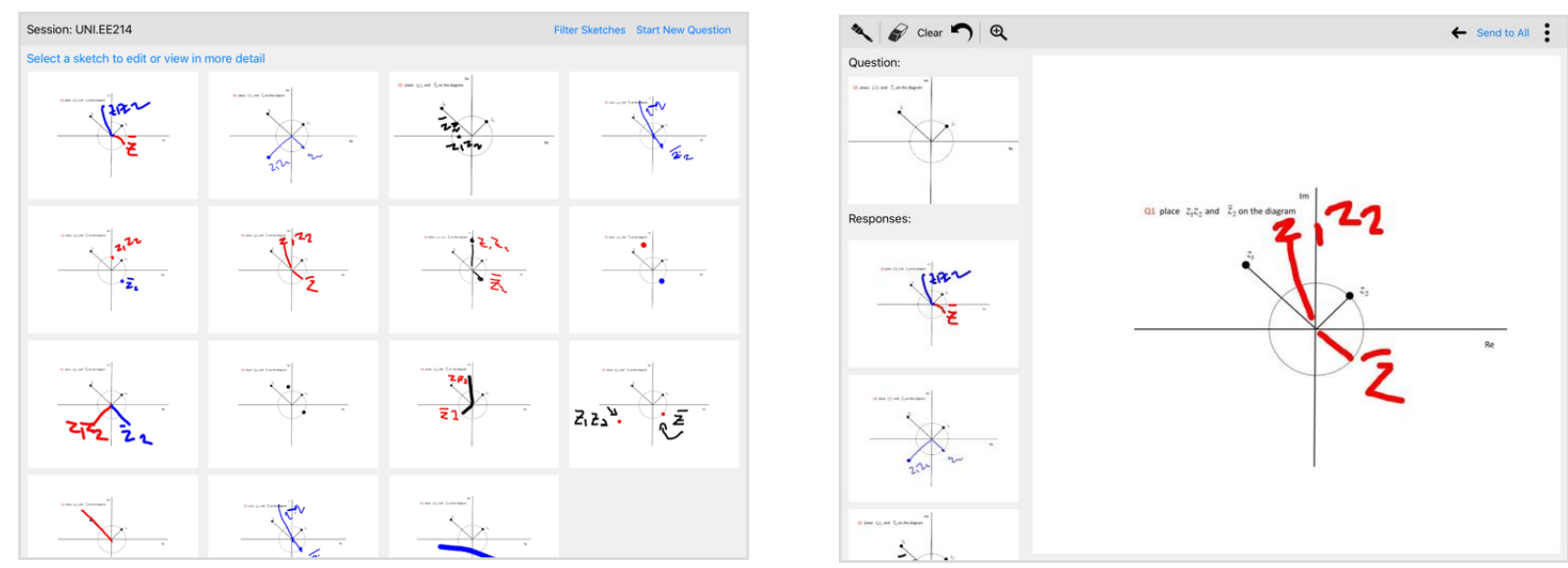

Figure 1. UniDoodle Viewing (left) and Editing (right) Teacher App.
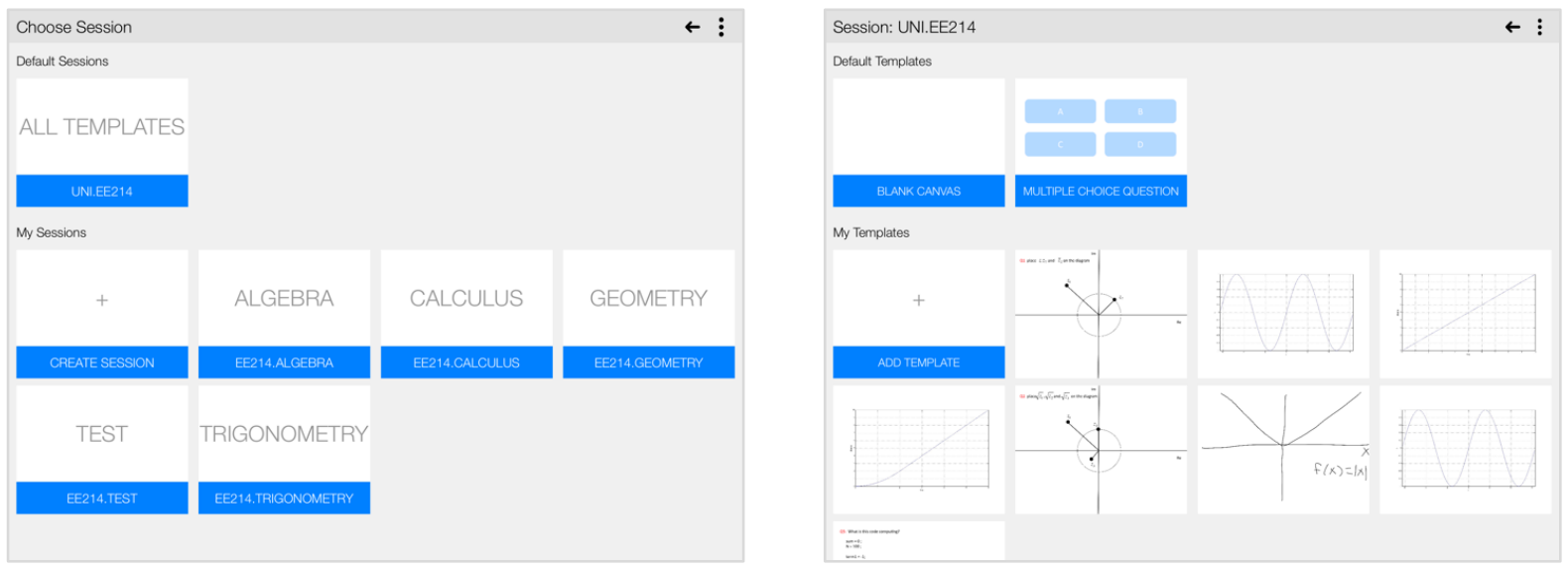

Figure 2. UniDoodle Session Management (left) and Template Development (right).

\section{Educational Context}

The UniDoodle response system was trialled in two first year modules which are offered by the School of Electronic Engineering in Dublin City University (DCU) to all their first year engineers. These consist of students taking the Common Entry, Electronic and Computer Engineering, Mechatronic Engineering, Mechanical and Manufacturing Engineering and Biomedical Engineering programmes. The modules were EM122 Engineering Mathematics II and EM114 Numerical Problem Solving for Engineers. The former covers traditional topics in calculus, matrices and complex numbers and had 165 registered students, while the latter had 151 registrations and 
introduces numerical techniques for approximately solving a range of practical problems based on the material covered previously in EM122 and elsewhere.

The UniDoodle system was trialled in 4 distinct 1-hour stand-alone sessions, between February and April 2016, as follows:

Session 1: Students had to investigate basic concepts in functional analysis. In particular students were asked to sketch, for example, trigonometric functions and investigate the difference between $a b s(\sin (x))$ and $\sin (\operatorname{abs}(x))$. They were also asked to sketch the derivative of a given function, based purely on the shape of its graph (no functional description, values or other information was given).

Session 2: Students had to explore more complicated topics in calculus, such as for example, identifying the derivative of $\operatorname{abs}(\sin (x))$ and $\sin (\operatorname{abs}(x))$ (two functions that had been explored in Session 1). In addition, applications of calculus were explored with students being presented with sketches of a particle's acceleration against time and asked to produce sketches of its speed and position. Importantly the functional description of acceleration was not given. This means that the student could not simply integrate to find the desired solutions and instead had to consider how the shape of the curve yields the required information.

Session 3: Students had to explore topics from complex numbers, namely the polar form of a complex number and its relationship with the number's position on the complex plane. Students were challenged to include on the diagram various powers or square roots of the given complex number. Again specific information about the arithmetic value of the complex number was not provided. The unit circle was provided for reference.

Session 4: This was a pre-exam session where the multiple choice questions from the previous year's exam paper was reviewed. Students primarily used the multiple choice facility on UniDoodle to submit their answers and engaged in peer-learning to review the submissions and decide, as a group, on the correct answers.

By way of example, the question shown in Figure 3 was used during Session 3. This question was designed to develop the students' knowledge of the use of the polar form of complex numbers, and the ease with which this form allows certain complex arithmetic operations to be carried out. The lecturer had noted how poorly students in previous years tended to perform on related questions in the annual module examination, and felt that UniDoodle would present an opportunity to address misconceptions in advance.

The objective of the question presented was to demonstrate that the square root operation can be estimated very easily by considering the polar form of the complex number. Students are deliberately not given any specific information about the numerical values of the three numbers. This deliberately forces students out of their comfort zone when working with complex numbers, and requires them to make estimations based on an appreciation of the meaning of the argument and modulus of a complex number, rather than blindly performing arithmetical calculations. Demonstrating conceptual understanding in this manner would be difficult without freeform input capabilities, such as those offered by UniDoodle. 


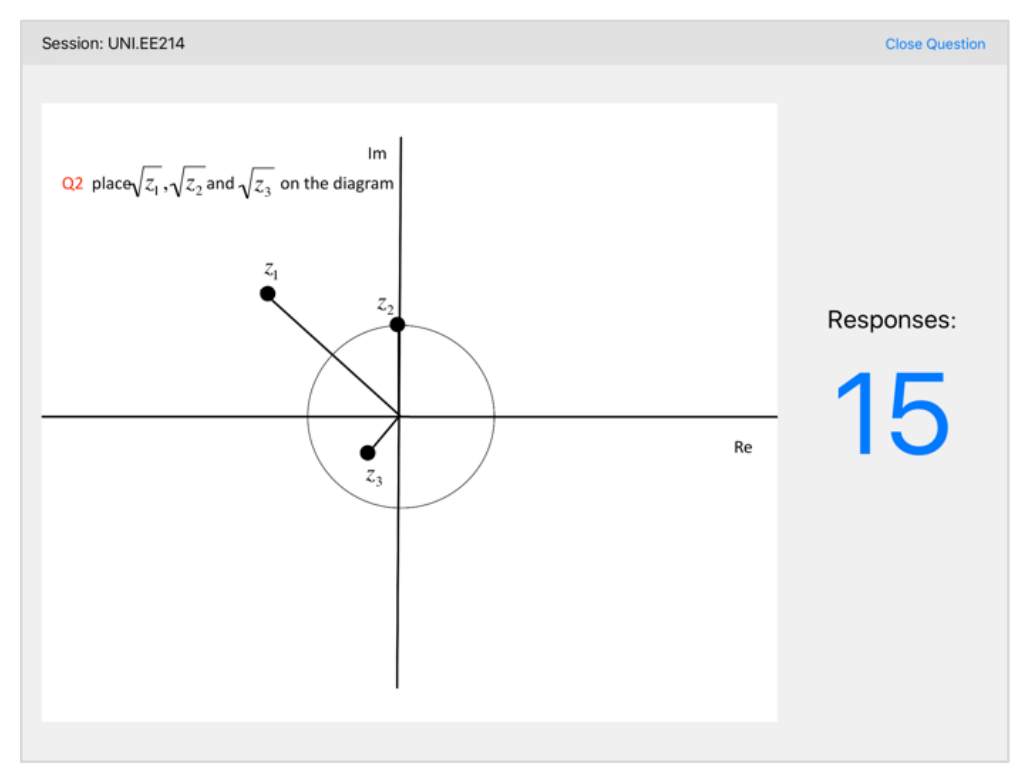

Figure 3. Sample question used in study.

\section{Evaluation Method}

At the end of the trial period, students were asked to complete detailed survey forms regarding their views on a range of aspects relating to the UniDoodle response system, including (i) usability, (ii) learning using the system and (iii) engagement in the classroom. In addition, the lecturer of the two modules was asked for his personal thoughts and opinion on the use of the UniDoodle system. It is worth noting that the lecturer had used the previous system and this allowed him to express relevant views on some of the new features of the UniDoodle system, as outlined in the previous section.

In total, 98 student survey forms were completed and returned at the end of the evaluation period. A selection of the student feedback is presented in Table 1 and Figure 4. Table 1 gives the average and standard deviation of the responses while Figure 4 shows a percentage breakdown of the responses per question.

\section{Results and Discussion}

In terms of engagement when using UniDoodle in class, students were generally positive about using the system, expressing that it provided an effective means of interacting with the lecturer. The feedback showed that the majority of students felt that UniDoodle helped them to be active in class, and over $90 \%$ of respondents felt that using the system was fun. As expected, most of the students (almost 93\%) noted that the anonymity of responses meant that they were more likely to respond to questions. This supports the sentiment echoed in much of the relevant research literature that inidicated anonymity would illicit a higher, and more honest, response from the class (Caldwell, 2007, Rowlett, 2010).

In using UniDoodle, students felt that both they themselves and the lecturer had experienced benefits regarding learning outcomes. The majority expressed that the use of the app allowed the lecturer to identify problem areas, and felt that feedback provided by the lecturer after completing a question helped to improve their understanding of concepts covered. They also stated that 
UniDoodle allowed them to measure their own performance and understanding, raised their awareness of what they did not know, and helped them to focus on what they should be learning.

The majority of students were also positive about the usability of the app, finding it easy to use and intuitive to navigate. Most students found it useful to be able to draw sketches using UniDoodle, although a few noted, via additional comments, that some of the drawing aspects could be improved. These included providing a keyboard input for text input, a thinner pen size option for neater sketching and the inclusion of a library of basic geometry shapes for convenience.

Table 1. Average and Standard Deviation of Student feedback regarding UniDoodle (1 to 5 represents strongly disagree, disagree, not sure, agree and strongly agree respectively).

\begin{tabular}{|l|c|c|}
\hline \multicolumn{1}{|c|}{ Statement } & $\begin{array}{c}\text { Average } \\
\text { Rating (1-5) }\end{array}$ & $\begin{array}{c}\text { Std. } \\
\text { dev. }\end{array}$ \\
\hline The use of UniDoodle helped me to be active in class. & 4.0 & 0.9 \\
\hline $\begin{array}{l}\text { The fact that my answers were anonymous encouraged me to submit my } \\
\text { responses in class. }\end{array}$ & 4.5 & 0.7 \\
\hline $\begin{array}{l}\text { UniDoodle makes me think more about the course material during my } \\
\text { lectures. }\end{array}$ & 3.5 & 1.1 \\
\hline I found it useful to be able to draw sketches with UniDoodle. & 4.0 & 0.8 \\
\hline I found this method of interaction between students and lecturer effective. & 4.1 & 0.8 \\
\hline I would recommend that the lecturer continue to use UniDoodle. & 3.7 & 1.1 \\
\hline The use of UniDoodle allows lecturers to identify problem areas. & 3.8 & 0.9 \\
\hline UniDoodle allows me to better understand key concepts & 3.6 & 0.8 \\
\hline $\begin{array}{l}\text { The feedback provided by the lecturer after completing a UniDoodle } \\
\text { question helped me focus on what I should be learning in the course. }\end{array}$ & 3.9 & 0.8 \\
\hline It was easy to use UniDoodle. & 4.4 & 0.8 \\
\hline I rarely had to seek help to use UniDoodle. & 4.2 & 0.9 \\
\hline
\end{tabular}

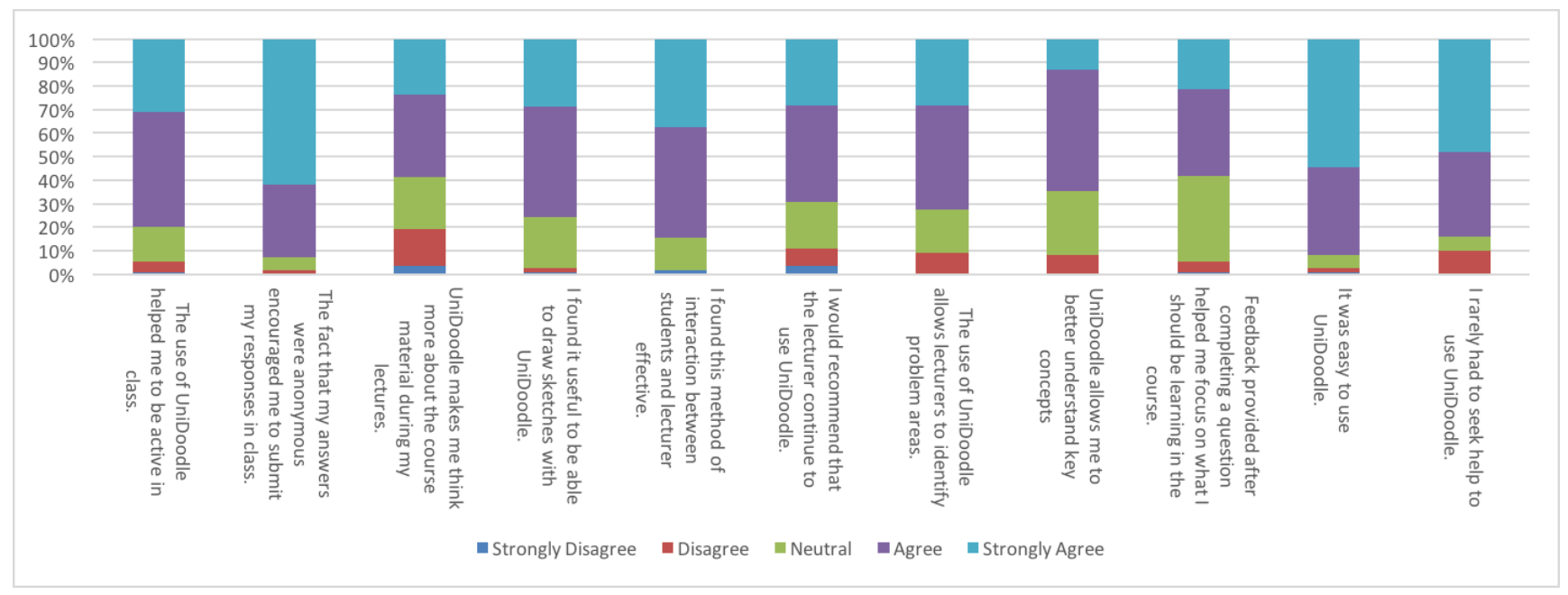

Figure 4. Detailed breakdown of student feedback regarding UniDoodle. 
When asked about the disadvantages of using UniDoodle, the most common sentiment was that having their phone out in class presented students with the opportunity to become distracted by other apps, with 54.5\% agreeing that they had been distracted. However, when asked to weigh the advantages and the disadvantages overall, for the use of UniDoodle in their module, the vast majority of students felt that UniDoodle was advantageous or very advantageous, with less than $10 \%$ disagreeing with this sentiment. In addition, almost $40 \%$ of respondents felt that using the app increased their confidence in their ability to complete the module successfully, while $45 \%$ were unsure. The reader is referred to Rowlett (2010) for an interesting discussion on the debatable merits of using response systems in a classroom environment, particularly in the context of improving learning outcomes. It should be noted, however, that this discussion predates the UniDoodle system and, so, it will be interesting to observe if UniDoodle can indeed improve learning outcomes. Such a study is beyond the scope of this paper.

The lecturer, who had used the previous version of the SRS, reported that UniDoodle constituted a great improvement. In particular, he found that the extension of the system to iPhones and iPads meant that coverage within the classroom was now effectively $100 \%$. He also noted that since the assessment was not summative, there was no issue with any students who did not have a device (or had forgotten one) as they could pair up with colleagues who did.

The lecturer also felt that the ability to filter responses was a great innovation. The lecturer started each session with a blank template and an invitation to the students to draw a picture of whatever they felt like. Ostensibly this was framed as an invitation to re-familiarise themselves with the basic functionality of the system, while in reality it was intended to quickly address and temper the temptation for students to abuse the system by submitting inappropriate images. The filter facility meant that such submissions could be removed without drawing attention to them and, in practice, students who were intent on disruption quickly realised that there would be no opportunity to do so.

The lecturer found that the template functionality offered a fantastic off-line way of producing good quality questions. However, noting that he occasionally asked on-the-spot questions in the classroom, he added that useful future innovations may include the ability to render simple geometrical figures, text and mathematical content in real-time. Interestingly, this echoed some of the comments given by the students themselves in relation to improving the sketch capabilities on UniDoodle.

As for its use in class, the lecturer found that the students greatly enjoyed using UniDoodle. "The shift from passive observers in a lecture to active participants is something that they really respond to." In addition, he felt that the anonymous nature of the interaction is vital, noting that it was "astounding that one can ask the simplest question in class to a resounding silence, while a question posed on UniDoodle can receive dozens of responses - many of which can be wrong, but at least have been volunteered".

Overall, the lecturer observed that the adoption of the UniDoodle system is very worthwhile, but that it was not a trivial undertaking. Devising exercises, which make appropriate use of the system, and test students' conceptual understanding of the problem, rather than their ability to merely solve a specific numerical example, requires time and practice.

Furthermore, given that he had to quickly examine up to 100 submissions for each question, he found that he had to carefully think about the types of answers that he was likely to receive, as well as common mistakes that were likely to present themselves. This would then allow him to react quickly in the classroom and identify submissions that were worthy of feedback. 


\section{Acknowledgements}

This project was funded by the National Forum for the Enhancement of Teaching \& Learning, Ireland.

\section{References}

Angelo, T. A. and Cross, K. P., 1993. Classroom Assessment Techniques: A Handbook for College Teachers. Jossey-Bass, San Francisco, CA.

Barber, M. and Njus, D., 2007. Clicker evolution: seeking intelligent design. The American Society for Cell Biology, 6, pp.1-20.

Blasco-Arcas, L., Buil, I., Hernández-Ortega, B. and Sese, F.J., 2013. Using clickers in class. The role of interactivity, active collaborative learning and engagement in learning performance. Computers and Education, 62(1), pp.102-110.

Bruff, D., 2009. Teaching with Classroom Response Systems: Creating Active Learning Environments. Jossey-Bass, San Francisco, CA.

Caldwell, J.E. 2007. Clickers in the large classroom: current research and best-practice tips. Life Sciences Education, 6, pp.9-20.

Fies, C. and Marshall, J., 2006. Classroom response systems: a review of the literature. Journal of Science Education and Technology, 15(1), pp.101-109.

Han, J.H. and Finkelstein, A., 2013. Understanding the effects of professors' pedagogical development with Clicker Assessment and Feedback technologies and the impact on students' engagement and learning in higher education. Computers and Education, 65, pp.64-76.

McLoone, S., Villing, R. and O'Keeffe, S., 2015. Using mobile touch devices to provide classroom assessment techniques. Int. Journal of Mobile Human Computer Interaction, 7(4), pp.1-15.

Miller, R.G., Ashar, B.H. and Getz, K.J., 2003. Evaluation of an audience response system for the continuing education of health professionals. Journal of Continuing Education in the Health Professions, 23, pp.109-115.

Moredich, C. and Moore, E., 2007. Engaging student through the use of classroom response systems. Nurse Educator, 32(3), pp.113-116.

Ramesh, N., 2011. Use of technology to enhance teaching and learning in mathematics and statistics. MSOR Connections, 11(1), pp. 34-36.

Reay, N.W., Bao, L., Pengfei, L., Warnakulasooriya R. and Baugh G., 2005. Towards an effective use of voting machines in physics lectures. American Journal of Physics, 73, pp.554-558.

Retkute, R., 2009. Exploring technology-based continuous assessment via electronic voting systems in mathematics and statistics. MSOR Connections, 9(1), pp. 24-28.

Roschelle, J., Penuel, W.R. and Abrahamson, A.L., 2004. Classroom response and communications systems; research review and theory. In Annual Meeting of the American Educational Research Association, San Diego, CA, pp.1-8.

Rowlett, P., 2010. Ask the Audience (yes, all of them). MSOR Connections, 10(1), pp. 3-5.

Sarason, Y. and Banbury, C., 2004. Active learning facilitated by using a game-show format or who doesn't want to be a millionaire? Journal of Management Education, 28, pp.509-519. 